\title{
Rapid loss of early antigen-presenting activity of lymph node dendritic cells against Ag85A protein following Mycobacterium bovis BCG infection
}

Zhengzhong $X u^{1}$, Aihong Xia', Xin $\mathrm{Li}^{1}$, Zhaocheng Zhu', Yechi Shen², Shanshan Jin², Tian Lan², Yuqing Xie², Han Wu' ${ }^{2}$ Chuang Meng ${ }^{1}$, Lin Sun ${ }^{1}$, Yuelan Yin², Xiang Chen ${ }^{1,3^{*}}$ and Xinan Jiao ${ }^{2,3^{*}}$

\begin{abstract}
Background: Control of Mycobacterium tuberculosis (Mtb) infection requires CD4 ${ }^{+}$T-cell responses and major histocompatibility complex class II (MHC II) presentation of Mtb antigens (Ags). Dendritic cells (DCs) are the most potent of the Ag-presenting cells and are central to the initiation of T-cell immune responses. Much research has indicated that DCs play an important role in anti-mycobacterial immune responses at early infection time points, but the kinetics of Ag presentation by these cells during these events are incompletely understood.

Results: In the present study, we evaluated in vivo dynamics of early Ag presentation by murine lymph-node (LN) DCs in response to Mycobacterium bovis bacillus Calmette-Guérin (BCG) Ag85A protein. Results showed that the early Ag-presenting activity of murine DCs induced by M. bovis BCG Ag85A protein in vivo was transient, appearing at $4 \mathrm{~h}$ and being barely detectable at $72 \mathrm{~h}$. The transcription levels of CIITA, MHC II and the expression of MHC II molecule on the cell surface increased following BCG infection. Moreover, BCG was found to survive within the inguinal LN DC pool, representing a continuing source of mycobacterial Ag85A protein, with which LN DCs formed Ag85A peptide-MHCIl complexes in vivo.
\end{abstract}

Conclusions: Our results demonstrate that a decrease in Ag85A peptide production as a result of the inhibition of Ag processing to is largely responsible for the short duration of Ag presentation by LN DCs during BCG infection in vivo.

Keywords: Ag-presenting activity, Dendritic cell, M. bovis BCG, Major histocompatibility complex class II, In vivo

\section{Background}

Tuberculosis (TB), caused by infection with Mycobacterium tuberculosis $(M t b)$, remains a major disease worldwide and is the leading infectious disease in terms of mortality, being responsible for an estimated 1.3 million deaths globally in 2016. Moreover, in the same year, there were an estimated 10.4 million new cases of active TB worldwide. Mycobacterium bovis bacillus Calmette-

\footnotetext{
* Correspondence: chenxiang@yzu.edu.cn; jiao@yzu.edu.cn

${ }^{1}$ Jiangsu Key Laboratory of Zoonosis, Yangzhou University, No. 48 Wenhui East Road, Yangzhou 225009, Jiangsu, China

${ }^{2}$ Key Laboratory of Prevention and Control of Biological Hazard Factors (Animal Origin) for Agrifood Safety and Quality, MOA of China, Yangzhou University, Yangzhou, China

Full list of author information is available at the end of the article
}

Guérin (BCG) is the only $\mathrm{TB}$ vaccine for humans in current use, but its efficacy is insufficient to prevent pulmonary TB in adults and reactivation of latent $M t b$ infection [1]. BCG vaccination mainly induces effector, rather than central, memory $\mathrm{T}$ cells, which are maintained for a shorter period, explaining the limited duration of protection afforded [2,3].

$\mathrm{CD} 4^{+}$T-cell responses and the production of interferon gamma (IFN- $\gamma$ ) are particularly important to the containment of $M t b$ infection [4, 5]. Dendritic cells (DCs) represent the bridge between the innate and adaptive immune responses and specifically strengthen the cellular immune response against mycobacterial infections $[6,7]$. Thus, the mechanisms involved in major 
histocompatibility complex class II (MHC II) antigen $(\mathrm{Ag})$ processing and presentation, which are required for $\mathrm{CD}^{+}{ }^{+}$T-cell activation, are crucial for controlling $M t b$ infection [8]. Much research has indicated that DCs play an important role in anti-mycobacterial immune responses in the early stages of infection, but little is known of the kinetics of Ag presentation by these cells soon after $M$. bovis BCG exposure. Indeed, efforts to understand the basis of protective immunity against $M t b$ have led us the examinntion of even earlier infection time points. We previously investigated the Ag-presenting cell (APC) functions of murine DCs during the first 2 weeks following intravenous administration of recombinant BCG (rBCG) expressing the Escherichia coli MalE protein as a reporter Ag [9]. However, this process has not yet been directly examined in lymph node (LN) DCs using an endogenous $M$. bovis BCG Ag.

In the present study, we evaluated the in vivo dynamics of early Ag presentation by murine inguinal LN DCs in response to $M$. bovis BCG. The results showed that the early Ag-presenting activity of murine DCs induced by $M$. bovis BCG Ag85A protein in vivo was transient and that the inhibition of $\mathrm{Ag}$ processing due to the decreased production of Ag85A peptide is the primary reason for the rapid loss of Ag85A peptide-MHC II complexes.

\section{Results}

\section{Stimulation of Ag85A-specific IFN- $p$ production in BCG- infected mice}

In order to evaluate the kinetics of the Ag85A-specific T-cell immune response to BCG infection, mononuclear cells isolated from BCG-immunized mice were stimulated in vitro with Ag85A peptide, Ag85A protein, or bovine purified protein (PPD), and concentrations of IFN- $\gamma$ in culture supernatants were measured. The result showed a significant increase in Ag85A-specific IFN- $\gamma$ production by inguinal LN mononuclear cells 3 days after BCG injection, with an even greater increase after
6 days (Fig. 1). Ag85A-specific T lymphocytes in both the spleen (Fig. 1a) and inguinal LN (Fig. 1b) produced high levels of IFN- $\gamma$ when stimulated with Ag85A, although IFN- $\gamma$ production was 10-fold higher in the LN group. This suggests that the Ag85A-specific T-cell immune response was initiated in the inguinal LN 6 days following BCG infection. Differences in IFN- $\gamma$ production in the murine spleen and LN may be a consequence of differences in the frequency of $\mathrm{T}$ cells among mononuclear cells.

\section{Dynamics of DC ag-presenting activity in vivo}

To investigate the dynamics of inguinal LN DC Ag presentation, we tested their capacity to stimulate DE10 T-cell hybridomas at several time points after subcutaneous injection of mice with BCG. The inguinal LN DCs (CD11 $\mathrm{c}^{\text {high }}$ ) were sorted by autoMACS with a purity of $94.7 \%$ (Fig. 2a). When mice were infected with BCG, LN DCs collected at early time points invoked a response from DE10 hybridomas, with IL-2 being detected following stimulation with those harvested $4 \mathrm{~h}$ post-injection, and the highest IL-2 production being observed in response to DCs from mice infected for $12 \mathrm{~h}$. However, IL-2 was only minimally produced in response to DCs from mice infected for $72 \mathrm{~h}$ (Fig. 2b). Interestingly, when mice were s.c. injected with heat-killed BCG, Ag-presenting activity markedly decreased from $12 \mathrm{~h}$ to $96 \mathrm{~h}$ post-injection, suggesting that live BCG is necessary for efficient $\mathrm{Ag}$ presentation by $\mathrm{DCs}$ in vivo (Fig. 2c). Together, these results indicate that the MHC II presentation of mycobacteria-derived peptides by inguinal LN DCs is only transient, with Ag85A peptide-MHC II complexes on the surfaces of inguinal LN DCs disappearing rapidly.

\section{Analysis of MHC II, CIITA and T-cell costimulatory molecules on DCs following BCG infection}

We measured the expression of cell surface markers involved in Ag presentation and T-cell interaction. Sorted
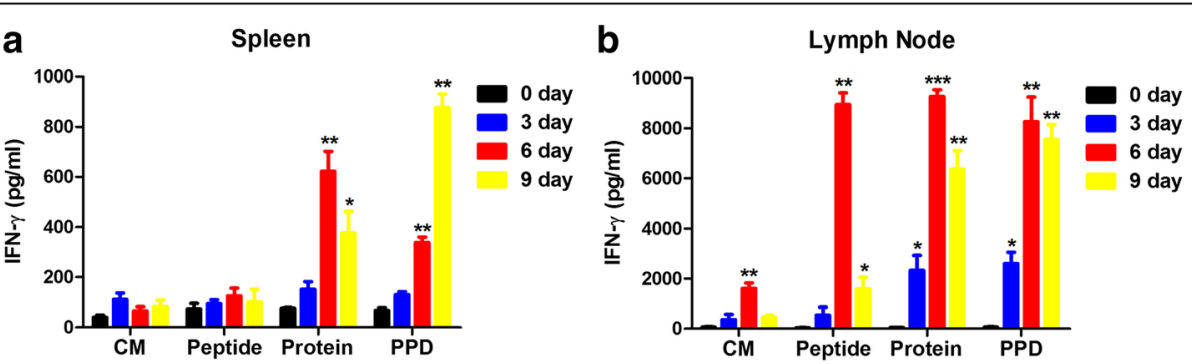

Fig. 1 Detection of IFN- $\gamma$ production following BCG infection. Four groups of C57BL/6 mice $(n=6)$ s.c. vaccinated with $1 \times 10^{8} \mathrm{CFU}$ BCG were sacrificed at different time points, and their spleens and inguinal LNs were removed. Increased IFN- $\gamma$ levels were detected in the culture supernatants of splenocytes (a) and inguinal LN cells (b). Results are representative of three independent experiments and presented as means \pm SEM. Statistical significance was determined using Student's $t$-test $\left({ }^{*} P<0.05,{ }^{* *} P<0.01,{ }^{* *} P<0.001\right)$. CM, culture medium 

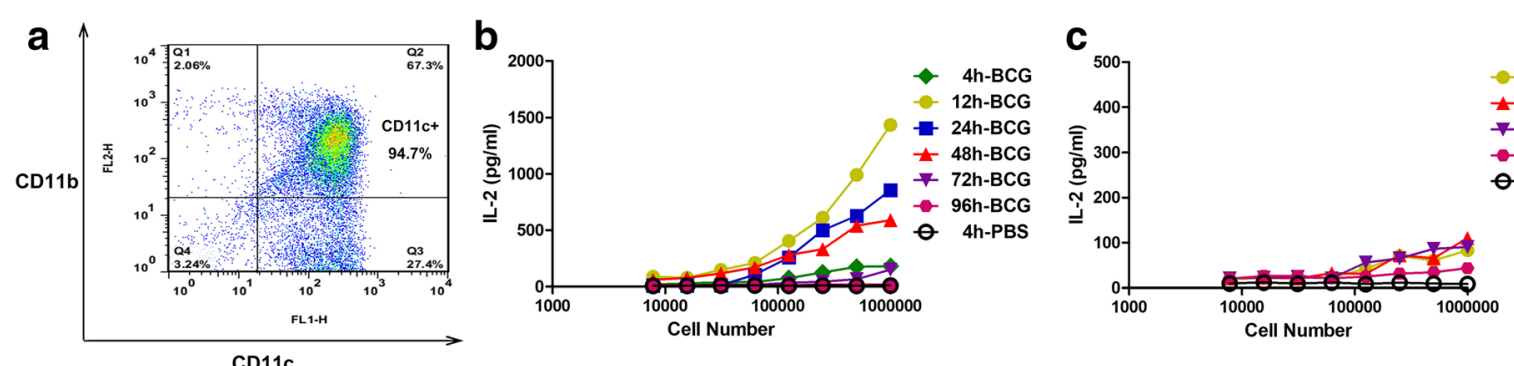

Fig. 2 Detection of murine LN DCs Ag-presenting activity ex vivo. Suspensions of inguinal LN cells from C57BL/6 mice were stained with antiCD11c MicroBeads and separated by autoMACS, resulting in a population of $94.7 \%$ CD11 $c^{+}$cells (a). To investigate the dynamics of LN DC Agpresenting activity, we harvested and sorted LN DCs from the inguinal LN at various time points groups after s.c. injection of mice $(n=6)$ with BCG or heat-killed BCG. Then, these cells were serially diluted and used to directly stimulate DE10 T-cell hybridomas. In vivo formation of Ag85A peptide-MHC complexes on LN DCs from mice injected with BCG (b) or heat-killed BCG (c) were estimated by measuring IL-2 production in DE10 T-cell hybridoma culture supernatants ex vivo. The experiment was repeated at least three times

inguinal LN DCs were stained with a panel of monoclonal antibodies (mAbs) to detect CD40, CD54, CD80, and CD86 by Flow Cytometry (FACS). No obvious regulation of CD40 or CD54 on DCs was observed during infection (Fig. 3a, b). High levels of CD80 and CD86 were noted at $12 \mathrm{~h}$, but the presence of these markers had decreased by $72 \mathrm{~h}$ and $96 \mathrm{~h}$ (Fig. 3c, d). These results indicate that inguinal LN DCs undergo functional activation in the early stages of BCG infection.

We next investigated transcription levels of MHC II and CIITA transcription in inguinal LN DCs during BCG infection using real-time PCR. MHC II expression was found to be increased by BCG infection at a relatively slow rate, while total CIITA transcription was rapidly induced, and expression of CIITA type I declined between 48 and $96 \mathrm{~h}$ (Fig. 3e). In addition, to evaluate the expression of MHC II molecules involved in $\mathrm{Ag}$ presentation, sorted inguinal LN DCs were stained with mAbs for FACS analysis. All sorted DCs demonstrated up-regulation of MHC II molecules following the initiation of infection (Fig. 3f). The fact that the transcription and expression of MHC II proteins on the cell surface did not decline following BCG infection suggests that the expression and trafficking of MHC class II molecules may be not associated with the rapid loss of Ag85A peptide-MHC II complexes. As a result, it can be deduced that LN DCs do not provide a continuous source of mycobacterial Ag85A peptides for the formation of peptideMHC II complexes.

\section{BCG infection kinetics of murine LN DCs}

In order to monitor the presence of BCG bacilli in inguinal LN DCs following s.c. infection of mice and thus evaluate the infection rate of these immune cells, rBCG-GFP cells were used in combination with FACS. As might be expected, $0.4 \%$ of LN DCs exhibited green fluorescence after $12 \mathrm{~h}$ of infection, and this figure had increased to $2 \%$ by $96 \mathrm{~h}$ post-injection (Fig. 4a). We next determined whether BCG survives and multiplies within DCs over the course of infection. CFUs appeared at $4 \mathrm{~h}$ and had increased significantly by $12 \mathrm{~h}$ after s.c. administration of BCG to mice, and numbers remained elevated until the end of the experiment (Fig. 4b). These results suggest that BCG infected the inguinal LN DC $12 \mathrm{~h}$ post-infection and that it survives within the inguinal LN DC pool, representing a continuing source of mycobacterial Ag85A protein, with which LN DCs can form Ag85A peptide-MHCII complexes in vivo.

\section{Discussion}

$\mathrm{CD} 4{ }^{+} \mathrm{T}$-cell responses and the production of IFN- $\gamma$ are particularly important to the containment of $M t b$ infection. In mice, between 1 and 3 weeks after initial infection, $M t b$-specific T cells appear in the lungs, IFN- $\gamma$ is expressed, and the bacterial burden is controlled [10]. Production of IFN- $\gamma$ by splenocytes in response to Ag restimulation is observed within 6 days after i.v. $M t b$ infection [11]. To determine when the T-cell response is initiated, we obtained splenocytes and inguinal LN cells from mice 3, 6, and 9 days after s.c. BCG injection. Inguinal LN cells collected 6 days after infection produced IFN- $\gamma$ in response to Ag85A restimulation. Thus, the T-cell immune response appears to have been initiated in the inguinal LN day 6 following BCG infection.

The mechanisms involved in MHC class II Ag processing and presentation, which are required for $\mathrm{CD} 4^{+}$ T-cell activation, are crucial for controlling $M t b$ infection. Previous research investigated the kinetics of Ag-presenting activity by harvesting spleens following i.v. administration of $\mathrm{rBCG}$ expressing the E. coli MalE protein as a reporter Ag. The formation of MalE peptide-MHC complexes in splenic DCs was detected at 2, 4, and $12 \mathrm{~h}$ after rBCG infection, while MalE was barely detectable at $48 \mathrm{~h}$ [9]. However, this process has not yet been directly examined in LN DCs and by using an endogenous $M$. bovis BCG Ag. To investigate the 


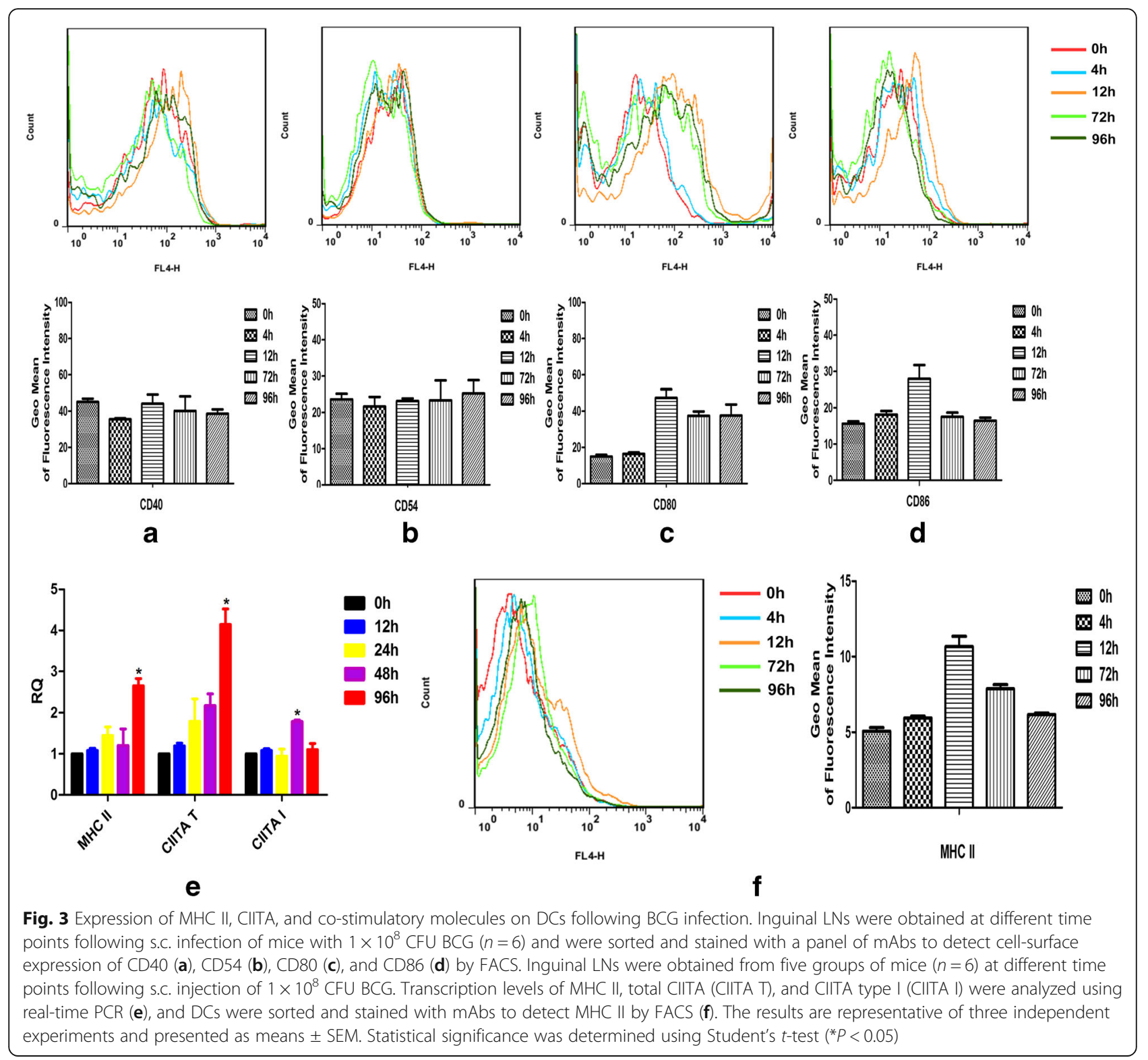

dynamics of LN DCs Ag presentation, we harvested and sorted these cells from inguinal LNs at several time points after s.c. injection of mice with Ag85A protein or BCG and tested their capacity to stimulate DE10 T-cell hybridomas, which are specific for an immunodominant Ag85A peptide. In this manner, in vivo formation of Ag85A peptide-MHC complexes on DCs from BCG-injected mice was detected by measuring IL-2 production in DE10 T-cell hybridoma culture supernatants ex vivo. Ag85A peptide-MHC complexes on LN DCs appeared rapidly after inoculation, with IL-2 production being detected in response to DCs collected $4 \mathrm{~h}$ after BCG infection and the highest production in response to those harvested at 12 h. By contrast, IL-2 levels following exposure to
DCs harvested $72 \mathrm{~h}$ after infection were barely detectable. Together, these results indicate that the MHC II presentation of mycobacteria-derived peptides by inguinal LN DCs is only transient, with Ag85A peptide-MHC II complexes on the surfaces of inguinal LN DCs disappearing rapidly. Some reports have shown that peptide-MHC complexes have a half-life of $25 \mathrm{~h}$ [12]. Thus, it can be concluded that the synthesis of Ag85A peptide-MHC II complexes on inguinal LN DCs was interfered.

Several reports have shown that $M t b$ and $M$. bovis inhibit intracellular processes associated with Ag presentation, including Ag processing, MHC class II expression, the trafficking of $\mathrm{MHC}$ class II molecules, and peptide-MHC class II binding [13, 14]. CIITA is the 

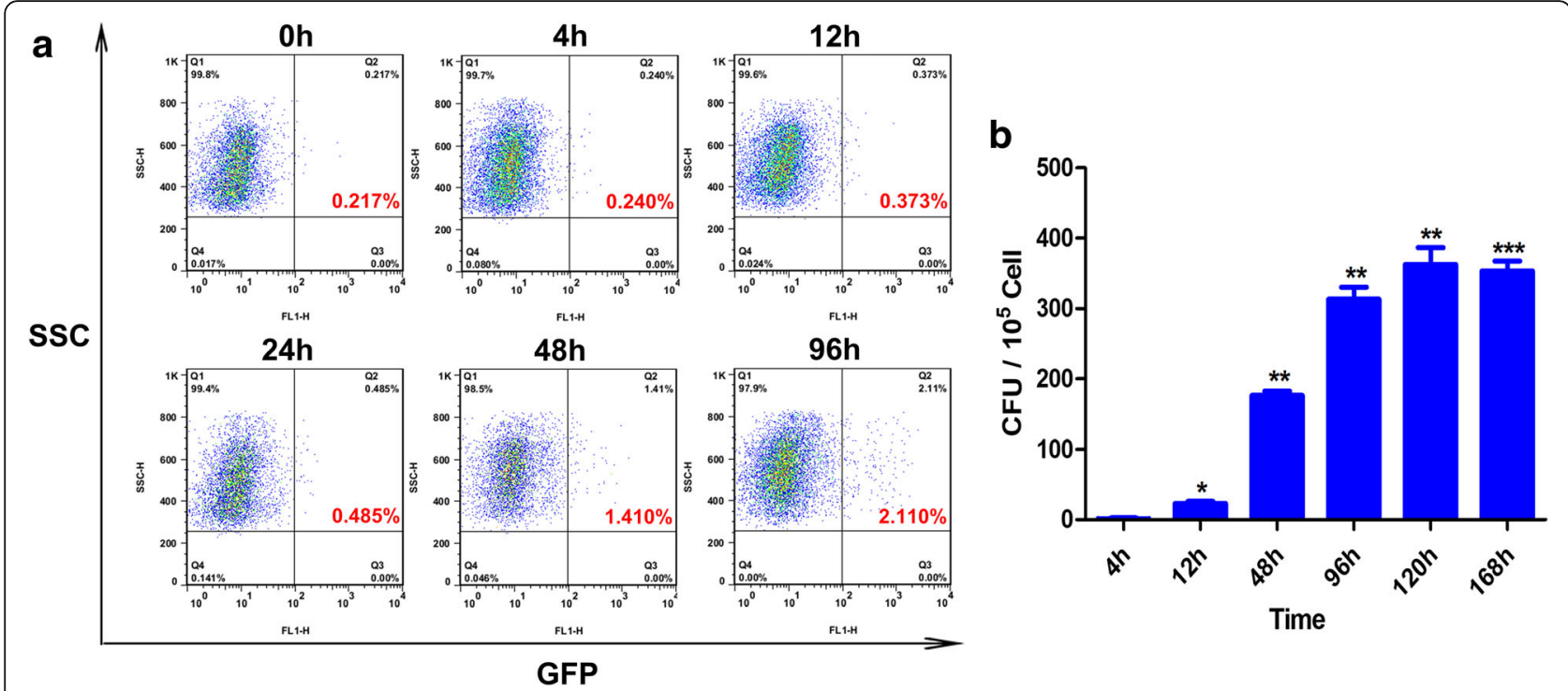

Fig. 4 BCG infection kinetics of murine LN DCs. Six groups of mice $(n=6)$ were s.c. injected with $1 \times 10^{8}$ CFU rBCG-GFP, and LN cells were harvested at different time points. DCs were sorted and analyzed for the presence of rBCG-GFP. Infection of murine LN DCs with BCG (a). Six groups of mice $(n=6)$ were s.c. injected with $1 \times 10^{8}$ CFU BCG, and BCG in DCs was quantified in CFUs by culturing on Middlebrook $7 \mathrm{H} 10$ agar (b). The results are representative of three independent experiments and presented as means \pm SEM. Statistical significance was determined using Student's t-test $\left({ }^{*} P<0.05,{ }^{* *} P<0.01,{ }^{* *} P<0.001\right)$

master transcriptional regulator of MHC class II molecules [15]. The transcription of CIITA itself is regulated by the three unique promoters pI, pIII, and pIV, which drive the expression of CIITA types I, III, and IV, respectively. pI is constitutively active in DCs [14]. In the current investigation, LN DCs exhibited the up-regulation of cell-surface MHC II molecules from 4 to $96 \mathrm{~h}$ following infection. Using real-time PCR, we analyzed the transcription levels of MHC II, total CIITA, and CIITA type I in DCs in response to BCG. Expression of MHC II was found to be induced by BCG infection relatively slowly, while total CIITA transcription was rapidly induced. This indicates that the transcription and expression of MHC II proteins on the cell surface did not declined following BCG infection, suggesting that the expression and trafficking of MHC class II molecules may be not associated with the rapid loss of Ag85A peptide-MHC II complexes. As a result, it can be deduced that LN DCs do not provide a continuing source of mycobacterial Ag85A peptides for the formation of peptide-MHC II complexes.

Considerable evidence shows that DCs can phagocytose mycobacteria and may be the first cells to encounter such pathogens, therefore, DCs are likely to be responsible for initiating the subsequent immune response. The survival of mycobacteria within DCs has been assessed previously in vitro using the BCG vaccine strain and virulent $M$. bovis, both of which were shown to be phagocytosed by DCs after $24 \mathrm{~h}$ of infection [16]. Mtb cells disseminate to draining LNs within 8 days following respiratory infection [17]. Approximately $2 \%$ of the splenic DC population (CD11 $\mathrm{c}^{+}$cells) was found to contain BCG at $4 \mathrm{~h}$ following i.v. infection [9]. In the present study, the presence of rBCG-GFP bacilli in inguinal LN DCs following s.c. inoculation of mice was monitored by FACS. As expected, the percentage of infected DCs increased to $2 \%$ after 96 h of infection. We then examined whether mycobacteria survive and multiply within DCs during infection. Following s.c. administration of BCG to mice, CFUs appeared at $4 \mathrm{~h}$, increased significantly by $12 \mathrm{~h}$, remaining elevated until the last time point. These results suggest that BCG survives within the inguinal LN DC pool, representing a continuing source of mycobacterial Ag85A protein with which LN DCs can form Ag85A peptide-MHCII complexes in vivo. Some reports have shown that live $M t b$ can alter phagosome maturation and decrease Ag processing, providing a mechanism for $M t b$ to evade immune surveillance and enhance its survival within the host [18-20]. Based on our findings, we conclude that the inhibition of $\mathrm{Ag}$ processing due to the reduced production of Ag85A peptide is the primary reason for the rapid loss of Ag85A peptide-MHC II complexes.

\section{Conclusions}

In the present study, we evaluated the in vivo dynamics of early Ag presentation by murine LN DCs in response to $M$. bovis BCG Ag85A protein. Our results showed that the early Ag-presenting activity of murine DCs induced by $M$. bovis BCG Ag85A protein in vivo was 
transient and that the inhibition of Ag processing induced by a decrease in the production of Ag85A peptide is the primary reason for the rapid loss of Ag85A peptide-MHC II complexes and the short duration of Ag presentation by LN DCs during BCG infection in vivo.

\section{Methods}

\section{Experimental animals}

Six-week-old female C57BL/6 mice were purchased from Vital River (Beijing, China). The mice were housed, handled, and immunized at our animal biosafety facilities, and all procedures were approved by the Institutional Animal Experimental Committee of Yangzhou University. All experiments were performed according to the national guidelines for animal welfare. The mice were euthanized by cervical dislocation under isoflurane, and spleens and inguinal LNs were collected for analysis.

\section{Bacterial strains and culture conditions}

M. bovis BCG Pasteur 1173P2 and rBCG expressing GFP (rBCG-GFP) were kindly provided by Dr. Xiaoming Zhang (Institut Pasteur of Shanghai, Chinese Academy of Sciences, Shanghai, China). Both strains were grown with gentle agitation $(80 \mathrm{rpm})$ in Middlebrook $7 \mathrm{H} 9$ medium (Difco, Detroit, MI, USA) supplemented with $0.05 \%$ Tween 80 and $10 \%$ albumin-dextrose-catalase (ADC) enrichment or on solid Middlebrook 7H10 medium (Difco) supplemented with $0.05 \%$ Tween 80 and $10 \%$ oleic-ADC enrichment.

\section{T-cell hybridoma and Ags}

MHC II-restricted DE10 T-cell hybridomas specific for the $M t b$ Ag85A peptide comprising amino acids 241 to 260 [21] were kindly provided by Dr. Claude Leclerc (Institut Pasteur, Paris, France). The Ag85A protein was constructed and expressed in our laboratory, and the Ag85A peptide (amino acids 241-260) was synthesized by SciLight Biotechnology (Beijing, China).

\section{Detection of IFN- $\gamma$ production following BCG infection} C57BL/6 mice were s.c. vaccinated with $1 \times 10^{8} \mathrm{CFU}$ BCG and sacrificed 3, 6, and 9 days later, at which point, spleens and inguinal LNs were removed aseptically and transferred to complete RPMI-1640 medium for preparation of single-cell suspensions. The mononuclear cells, isolated using Histopaque 1083 (Sigma, St. Louis, MO, USA), were seeded at $1 \times 10^{6}$ cells/well in 96-well plates containing complete RPMI-1640 medium. They were subsequently stimulated with $10 \mu \mathrm{g} / \mathrm{ml} \mathrm{Ag85A}$ peptide, $10 \mu \mathrm{g} / \mathrm{ml} \mathrm{Ag85A}$ protein, or $5 \mu \mathrm{g} / \mathrm{ml}$ bovine PPD (Prionics, Schlieren, Switzerland) and incubated at $37{ }^{\circ} \mathrm{C}$ in an atmosphere of $5 \% \mathrm{CO}_{2}$ in air. Supernatants were then harvested at $48 \mathrm{~h}$ post-stimulation, frozen, and later tested for IFN- $\gamma$ concentration by sandwich enzyme-linked immunosorbent assay (ELISA, BD Biosciences, Franklin Lakes, NJ, USA).

\section{Ag presentation assay}

C57BL/6 mice were s.c. injected with $1 \times 10^{8}$ CFU BCG or heat-killed BCG in $200 \mu \mathrm{l}$ PBS or with PBS alone. Mice were sacrificed at various time points, and their inguinal LNs removed and perfused with $400 \mathrm{U} / \mathrm{ml}$ collagenase type IV (Invitrogen, Carlsbad, CA, USA) containing $50 \mu \mathrm{g} / \mathrm{ml}$ DNase I (Invitrogen). Single LN-cell suspensions were prepared, and DCs were sorted with an autoMACS separator (Miltenyi Biotec, Bergisch Gladbach, Germany) using CD11c as a cell marker. Specifically, LN cells were first incubated with anti-CD11c MicroBeads (Miltenyi Biotec) before autoMACS separation, resulting in a population of CD11 $c^{\text {high }}$ cells (DCs). The purity of these murine LN DCs was then analyzed using a FACSCalibur instrument (BD Biosciences). For the ex vivo Ag presentation assay itself, the purified LN DCs were transferred to 96-well microplates and serially diluted in complete RPMI-1640 medium. DE10 T-cell hybridomas at a density of $1 \times 10^{5} /$ well were then added, and after incubation for $24 \mathrm{~h}$, supernatants were collected, frozen, and later tested for IL-2 content by sandwich ELISA (BD Biosciences).

\section{Cell phenotype analysis}

C57BL/6 mice were s.c. injected with $1 \times 10^{8}$ CFU BCG in $200 \mu \mathrm{l}$ PBS or with PBS alone. The mice were sacrificed after various periods for the preparation of single inguinal LN-cell suspensions and sorting of DCs by autoMACS. FITC-conjugated anti-CD11c, and biotinylated anti-I-Ad, anti-CD40, anti-CD54, anti-CD80, and anti-CD86 antibodies were used to label cells. Allophycocyanin-conjugated streptavidin was employed to visualize biotin conjugates. A FACSCalibur and FlowJo software (FlowJo LLC, Ashland, OR, USA) were then used for multicolor staining analysis of the labeled cells. DCs were sorted using the autoMACS system before being pelleted and resuspended in lysis buffer. Cellular RNA was purified with an RNeasy kit (Qiagen, Valencia, CA, USA) according to the manufacturer's instructions, and total RNA was reverse-transcribed into cDNA using SuperScript reverse transcriptase (Thermo Fisher Scientific, Waltham, MA, USA).

\section{In vivo infection assay}

C57BL/6 mice were s.c. injected with $1 \times 10^{8}$ CFU BCG or rBCG-GFP in $200 \mu \mathrm{l} \mathrm{PBS}$ or with PBS alone. Mice were sacrificed at various time points, and single inguinal LNs were removed aseptically and transferred to complete RPMI-1640 medium. Single-cell suspensions were prepared, and DCs were sorted with an autoMACS 
separator as above. The percentage of DCs infected with rBCG-GFP was analyzed using a FACSCalibur instrument and FlowJo software. BCG-infected DCs were pelleted and resuspended in lysis buffer. Ten-fold serial dilutions of these suspensions were then plated on solid Middlebrook 7H10 medium, and colonies were counted after incubation at $37^{\circ} \mathrm{C}$ for $2-3$ weeks.

\section{Statistical analysis}

All data are expressed as means \pm SE. Statistical analysis was performed by Student's $t$-test using GraphPad Prism software. $P$ values $<0.05$ were considered statistically significant.

\section{Abbreviations \\ ADC: Albumin-dextrose-catalase; APC: Antigen-presenting cell; BCG: Bacillus Calmette-Guérin; CIITA: MHC class II transactivator; DC: Dendritic cell; LN: Lymph node; MHC II: Major histocompatibility complex class II; Mtb: Mycobacterium tuberculosis; PPD: Purified protein derivative; rBCG: Recombinant BCG; TB: Tuberculosis}

\section{Acknowledgements}

The authors are grateful to Dr. Claude Leclerc (Institut Pasteur, Paris, France) and Dr. Xiaoming Zhang (Institut Pasteur of Shanghai, Chinese Academy of Sciences, Shanghai, China).

\section{Funding}

This work was supported in part by the National Key Research and Development Program of China (2017YFD0500300), the National Natural Science Foundation of China (31602031), the Science and Technology Program of Jiangsu (BK20160466, 16KJB230003, BK20171285 and BK20170493), and the Qinglan Project, Six Talent Peaks Project and Priority Academic Development Program of Jiangsu Higher Education Institutions (PADP).

\section{Availability of data and materials}

All data generated or analyzed during the current study are available from the corresponding author on reasonable request.

\section{Authors' contributions}

$Z Z X, X C$ and $X A$ J designed the experiments. ZZ X, AH X, XL, ZC Z, YC S, SS $J, T L, Y Q X, H W$, and $C M$ performed the experiments and analyzed the data. $L S, Y L Y, X C$, and $X A J$ contributed reagents/materials/analysis tools. $Z Z X, X C$, and $X A J$ wrote and revised the paper. All authors read and approved the final manuscript.

\section{Ethics approval and consent to participate}

The mice were housed, handled, and immunized at our animal biosafety facilities, and all procedures were approved by the Institutional Animal Experimental Committee of Yangzhou University. All experiments were performed according to the national guidelines for animal welfare.

\section{Consent for publication}

Not applicable.

\section{Competing interests}

The authors declare that they have no competing interests.

\section{Publisher's Note}

Springer Nature remains neutral with regard to jurisdictional claims in published maps and institutional affiliations.

\section{Author details}

'Jiangsu Key Laboratory of Zoonosis, Yangzhou University, No. 48 Wenhui East Road, Yangzhou 225009, Jiangsu, China. ${ }^{2}$ Key Laboratory of Prevention and Control of Biological Hazard Factors (Animal Origin) for Agrifood Safety and Quality, MOA of China, Yangzhou University, Yangzhou, China. ${ }^{3}$ Jiangsu
Co-Innovation Center for Prevention and Control of Important Animal Infectious Diseases and Zoonoses, Yangzhou University, Yangzhou, China.

Received: 27 March 2018 Accepted: 18 June 2018

Published online: 25 June 2018

\section{References}

1. Aguilo N, Gonzalo-Asensio J, Alvarez-Arguedas S, Marinova D, AB Gomez S, Uranga R, Spallek M, Singh M, Audran R, Spertini F, et al. Reactogenicity to major tuberculosis antigens absent in BCG is linked to improved protection against Mycobacterium tuberculosis. Nat Commun. 2017;8:16085.

2. Kaveh DA, Bachy VS, Hewinson RG, Hogarth PJ. Systemic BCG immunization induces persistent lung mucosal multifunctional CD4 T(EM) cells which expand following virulent mycobacterial challenge. PLoS One. 2011;6: e21566.

3. Vogelzang A, Perdomo C, Zedler U, Kuhlmann S, Hurwitz R, Gengenbacher M, Kaufmann SH. Central memory CD4+ T cells are responsible for the recombinant Bacillus Calmette-Guerin DeltaureC::hly vaccine's superior protection against tuberculosis. J Infect Dis. 2014;210: 1928-37.

4. Kaufmann E, Spohr C, Battenfeld S, De Paepe D, Holzhauser T, Balks E, Homolka S, Reiling N, Gilleron M, Bastian M. BCG vaccination induces robust CD4+ T cell responses to Mycobacterium tuberculosis complexspecific Lipopeptides in Guinea pigs. J Immunol. 2016;196:2723-32.

5. Lindestam Arlehamn CS, McKinney DM, Carpenter C, Paul S, Rozot V, Makgotlho E, Gregg Y, van Rooyen M, Ernst JD, Hatherill M, et al. A quantitative analysis of complexity of human pathogen-specific CD4 T cell responses in healthy $M$. Tuberculosis infected south Africans. PLoS Pathog. 2016;12:e1005760.

6. Griffiths KL, Ahmed M, Das S, Gopal R, Horne W, Connell TD, Moynihan KD, Kolls JK, Irvine DJ, Artyomov MN, et al. Targeting dendritic cells to accelerate T-cell activation overcomes a bottleneck in tuberculosis vaccine efficacy. Nat Commun. 2016;7:13894.

7. Lozza L, Farinacci M, Bechtle M, Staber M, Zedler U, Baiocchini A, Del Nonno F, Kaufmann SH. Communication between human dendritic cell subsets in tuberculosis: requirements for naive CD4(+) T cell stimulation. Front Immunol. 2014:5:324.

8. Torres M, Ramachandra L, Rojas RE, Bobadilla K, Thomas J, Canaday DH, Harding CV, Boom WH. Role of phagosomes and major histocompatibility complex class II (MHC-II) compartment in MHC-II antigen processing of Mycobacterium tuberculosis in human macrophages. Infect Immun. 2006: 74:1621-30.

9. Jiao X, Lo-Man R, Guermonprez P, Fiette L, Deriaud E, Burgaud S, Gicquel B, Winter N, Leclerc C. Dendritic cells are host cells for mycobacteria in vivo that trigger innate and acquired immunity. J Immunol. 2002;168:1294-301.

10. Harding CV, Boom WH. Regulation of antigen presentation by Mycobacterium tuberculosis: a role for toll-like receptors. Nat Rev Microbiol. 2010:8:296-307.

11. Tian T, Woodworth J, Skold M, Behar SM. In vivo depletion of CD11C+ cells delays the CD4+ T cell response to Mycobacterium tuberculosis and exacerbates the outcome of infection. J Immunol. 2005;175:3268-72.

12. Lanzavecchia A, Reid PA, Watts C. Irreversible association of peptides with class II MHC molecules in living cells. Nature. 1992;357:249-52.

13. Chang ST, Linderman JJ, Kirschner DE. Multiple mechanisms allow Mycobacterium tuberculosis to continuously inhibit MHC class IImediated antigen presentation by macrophages. Proc Natl Acad Sci U S A. $2005 ; 102: 4530-5$

14. Srivastava S, Grace PS, Ernst JD. Antigen export reduces antigen presentation and limits T cell control of M. Tuberculosis. Cell Host Microbe. 2016;19:44-54.

15. Ghorpade DS, Holla S, Sinha AY, Alagesan SK, Balaji KN. Nitric oxide and KLF4 protein epigenetically modify class II transactivator to repress major histocompatibility complex II expression during Mycobacterium bovis bacillus Calmette-Guerin infection. J Biol Chem. 2013;288:20592-606.

16. Hope JC, Thom ML, McCormick PA, Howard CJ. Interaction of antigen presenting cells with mycobacteria. Vet Immunol Immunopathol. 2004; 100:187-95

17. Chackerian AA, Alt JM, Perera TV, Dascher CC, Behar SM. Dissemination of Mycobacterium tuberculosis is influenced by host factors and precedes the initiation of T-cell immunity. Infect Immun. 2002;70:4501-9. 
18. Ramachandra L, Noss E, Boom WH, Harding CV. Processing of Mycobacterium tuberculosis antigen 85B involves intraphagosomal formation of peptide-major histocompatibility complex II complexes and is inhibited by live bacilli that decrease phagosome maturation. J Exp Med. 2001;194:1421-32.

19. Sendide K, Deghmane AE, Pechkovsky D, Av-Gay Y, Talal A, Hmama Z. Mycobacterium bovis BCG attenuates surface expression of mature class II molecules through IL-10-dependent inhibition of cathepsin S. J Immunol. 2005;175:5324-32

20. Singh CR, Moulton RA, Armitige LY, Bidani A, Snuggs M, Dhandayuthapani S, Hunter RL, Jagannath C. Processing and presentation of a mycobacterial antigen $85 \mathrm{~B}$ epitope by murine macrophages is dependent on the phagosomal acquisition of vacuolar proton ATPase and in situ activation of cathepsin D. J Immunol. 2006;177:3250-9.

21. Johansen P, Fettelschoss A, Amstutz B, Selchow P, Waeckerle-Men Y, Keller P, Deretic V, Held L, Kundig TM, Bottger EC, et al. Relief from Zmp1mediated arrest of phagosome maturation is associated with facilitated presentation and enhanced immunogenicity of mycobacterial antigens. Clin Vaccine Immunol. 2011;18:907-13.

Ready to submit your research? Choose BMC and benefit from:

- fast, convenient online submission

- thorough peer review by experienced researchers in your field

- rapid publication on acceptance

- support for research data, including large and complex data types

- gold Open Access which fosters wider collaboration and increased citations

- maximum visibility for your research: over $100 \mathrm{M}$ website views per year

At BMC, research is always in progress.

Learn more biomedcentral.com/submissions 